\title{
Characterization of Nadir Echoes in Multiple- Elevation-Beam SAR With Constant and Variable Pulse Repetition Interval
}

\author{
Michelangelo Villano $^{\circledR}$, Senior Member, IEEE, and Maxwell Nogueira Peixoto ${ }^{\circledR}$
}

\begin{abstract}
Multiple-elevation-beam synthetic aperture radar (SAR) is a concept based on digital beamforming (DBF) in elevation and simultaneous recording of the echoes of multiple transmitted pulses. It enables high-resolution imaging of wide areas and is therefore ideal for the systematic observation of dynamic processes on the Earth's surface. Furthermore, if the pulse repetition interval (PRI) is continuously varied (staggered SAR), it is possible to map a wide continuous swath rather than multiple subswaths separated by "blind" ranges. Within the design of multiple-elevation-beam SAR, however, it is fundamental to consider how nadir echoes affect the mapping capabilities of systems with constant PRI and the image quality of staggered SAR systems, where nadir echoes are intrinsically smeared due to the PRI variation. This article addresses the characterization of nadir echoes in multiple-elevation-beam SAR with constant and variable PRI by presenting a parametric model for the nadir echo profile based on real radar measurements, a formulation of the nadir echo location and smearing in staggered SAR, and realistic simulations based on TerraSAR-X data, which show that nadir echo are likely to be barely visible in staggered SAR images. The results of this work are relevant to both the design of future SAR systems and the interpretation of the acquired data.
\end{abstract}

Index Terms-Digital beamforming (DBF), high-resolution wide-swath (HRWS) imaging, multiple-elevation-beam SAR, nadir echo, NASA-ISRO SAR (NISAR), staggered SAR, synthetic aperture radar (SAR), Tandem-L.

\section{INTRODUCTION}

$\mathbf{S}$ YNTHETIC aperture radar (SAR) is a widely-used remote-sensing technology, capable of providing high-resolution images independent of weather conditions and sunlight illumination [1]. However, conventional SAR systems are intrinsically limited, in that a wide swath can only be mapped at the expense of a degraded azimuth resolution, i.e., by increasing the pulse repetition interval (PRI). This limitation can be overcome using systems with multiple receive apertures, displaced in along-track, which simultaneously acquire multiple samples for each transmitted pulse [2]-[6]. These systems, however, require a very long antenna to map a wide swath.

If a relatively short antenna with a single aperture in along-track is available, an attractive solution to map a wide swath is given by SAR systems that exploit a wide beam

Manuscript received July 19, 2021; accepted August 3, 2021. (Corresponding author: Michelangelo Villano.)

Michelangelo Villano is with German Aerospace Center (DLR), Microwaves and Radar Institute Oberpfaffenhofen, 82234 Wessling, Germany (e-mail: michelangelo.villano@dlr.de).

Maxwell Nogueira Peixoto was with German Aerospace Center (DLR), Microwaves and Radar Institute Oberpfaffenhofen, 82234 Wessling, Germany.

Digital Object Identifier 10.1109/TGRS.2021.3103266 illuminator on transmit and digital beamforming (DBF) in elevation to form multiple receive beams, which follow the directions of arrival of the radar echoes of multiple transmitted pulses and can therefore simultaneously image multiple subswaths [7], [8]. If such systems are operated with a constant PRI, "blind" ranges will be present between the multiple imaged subswaths, as the radar cannot receive while it is transmitting. In contrast, if the PRI is continuously varied and data are sufficiently oversampled in azimuth-the overall concept is referred to as staggered SAR - it is possible to get rid of blind ranges and map a wide continuous swath [9]-[12]. The multiple-elevation-beam SAR concept is well established and currently being considered as the baseline acquisition mode for the Tandem-L mission proposal and the NASA-ISRO SAR (NISAR) and ALOS-4 missions [13]-[17]. It furthermore represents an attractive solution for the future SAR missions of European Space Agency's Copernicus Program [18].

Within the design of multiple-elevation-beam SAR systems, it is fundamental to consider the impact of nadir echoes in terms of both mapping limitation, in case nadir echoes are avoided through PRI selection in the constant PRI case, and image quality degradation, in case nadir echoes are present and superimpose on the underlying scene for the variable PRI case, for which a comprehensive analysis is still missing. In the latter case, a thorough understanding of the nadir echo signature allows providing recommendations for the design of the staggered SAR system, e.g., regarding the selection of the PRI sequence.

The article is organized as follows. Section II presents a parametric model for nadir echo based on real radar measurements. Section III discusses the mapping limitations imposed by nadir echoes on a multiple-elevation-beam SAR with constant PRI, whereas Section IV provides an analytical formulation of nadir echo location and smearing in multiple-elevation-beam SAR with variable PRI. In Section V, the impact of nadir echoes on SAR image quality is assessed through simulations based on real TerraSAR-X data and conclusion is drawn in Section VI.

\section{Parametric Model for Nadir Echoes}

Delay/Doppler radar altimeters allow retrieving important geophysical parameters and several models have been proposed through the years for the received echoes [19]-[22]. Nadir echoes in SAR images result from succeeding pulse echoes that are scattered back from the nadir, i.e., the point 
(a)

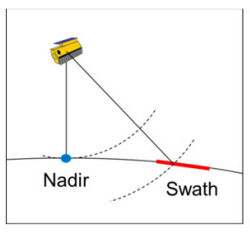

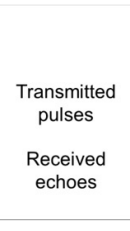

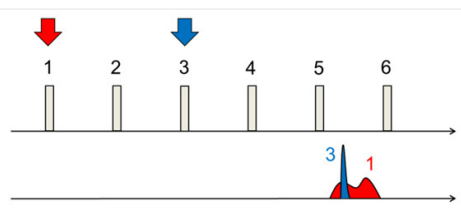

(b)
Fig. 1. (a) Typical geometry of an SAR. The imaged swath is highlighted in red and the nadir in blue. (b) Transmitted pulses (top) and nadir echo of transmitted pulse no. 3 (in blue) superimposed on swath echo of transmitted pulse no. 1 (in red) (bottom).
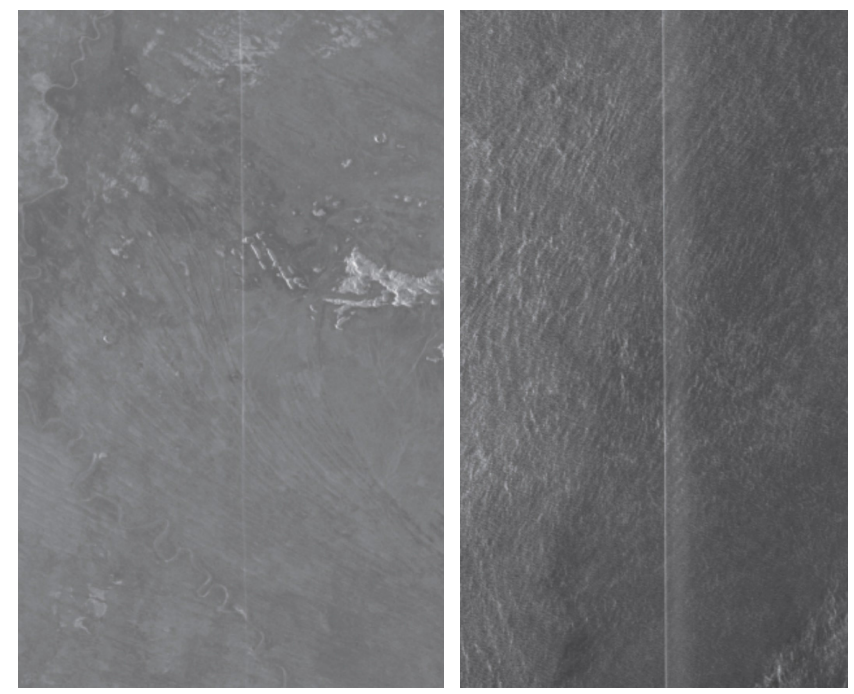

Fig. 2. Example of SAR images, acquired by the TerraSAR-X satellite over Australia (left) and the Indian Ocean (right), where nadir echoes appear as a bright vertical stripe in the middle of the image. The horizontal and vertical axes represent slant range and azimuth, respectively.

with the closest distance to the radar, and come back at the radar simultaneously with the echoes from the imaged swath and represent a disturbance. Fig. 1(a) shows the typical side-looking geometry of an SAR, where the imaged swath is highlighted in red and the nadir in blue. In Fig. 1(b) the sequence of transmitted pulses is displayed, where the nadir echo of transmitted pulse no. 3 (in blue) superimposes on the swath echo of transmitted pulse no. 1 (in red).

Although the radar antenna is designed to limit the energy transmitted to and received from the nadir direction, due to the smaller range and the specific characteristics of the scattering process (specular reflection), the nadir echo may be even stronger than the desired one and may therefore significantly affect the quality of the SAR image [23]. Fig. 2 shows two SAR images collected during dedicated TerraSAR-X acquisitions over Australia and the Indian Ocean, respectively, where nadir echoes appear as a bright stripe [24].

The two aforementioned TerraSAR-X acquisitions were carried out to investigate in more detail the properties of nadir echoes and can be exploited to derive a simple, parametric model for the nadir echo that consists of a backscatter profile and an azimuth autocorrelation function. This model is therefore not based on the electromagnetic theory of scattering, but simply on a fit of a parameterized function to real radar measurements. In particular, the dataset acquired over the Indian Ocean is used, as the lower backscatter of the underlying scene allows a better analysis of the falling edge of the nadir echo, as also apparent from the visual comparison of the two SAR images in Fig. 2.

The model is derived from range-compressed data (Fig. 3(a) shows a few hundred azimuth lines, where the nadir echo signature is visible) by aligning all range lines to compensate for the tilt due to the height change during the acquisition [see Fig. 3(b)]. The backscatter profile is then obtained by averaging across azimuth to remove the speckle. Fig. 3(c) shows a range line of the aforementioned range-compressed data [a zoom around the echo peak is displayed in Fig. 3(d)], where the effect of speckle is visible, whereas Fig. 3(e) shows the backscatter profile obtained after aligning and averaging all range lines of the dataset [a zoom around the echo peak is displayed in Fig. 3(f)].

The nadir echo backscatter profile $\sigma^{0}(t)$, where $t$ denotes the range (or fast) time, can be modeled through the continuous and differentiable piecewise function

$$
\sigma^{0}(t)=\left\{\begin{array}{l}
0, t<t_{\max }-\tau_{\text {rise }} \\
\sigma_{\max }^{0} \cdot 2^{\alpha-1}\left(\frac{t-t_{\text {max }}}{\tau_{\text {rise }}}+1\right)^{\alpha}, \\
t_{\max }-\tau_{\text {rise }} \leq t<t_{\max }-\tau_{\text {rise }} / 2 \\
\sigma_{\max }^{0}\left[1-2^{\alpha-1}\left(\frac{t_{\max }-t}{\tau_{\text {rise }}}\right)^{\alpha}\right], \\
t_{\max }-\tau_{\text {rise }} / 2 \leq t<t_{\max } \\
\sigma_{\max }^{0}\left[(1-\beta)\left(\frac{t-t_{\max }}{\tau_{\text {fall }}}\right)^{3}+\right. \\
\left.\left(\beta-\frac{3}{2}\right)\left(\frac{t-t_{\max }}{\tau_{\text {fall }}}\right)^{2}+1\right], \\
t_{\max } \leq t<t_{\max }+\tau_{\text {fall }} \\
\frac{\sigma_{\max }^{0}}{2\left(\frac{t-t_{\max }}{\tau_{\text {fall }}}\right)^{2 \beta}}, t \geq t_{\max }+\tau_{\text {fall }}
\end{array}\right.
$$

where $t_{\max }$ is the range time at which the backscatter is maximum, $\sigma_{\max }^{0}$ is the maximum backscatter, i.e., $\sigma_{\max }^{0}=\sigma^{0}\left(t_{\max }\right)$, $\tau_{\text {rise }}$ is the rising time, defined as the time interval between the start of the echo and its maximum, $\alpha, \alpha>1$, is a parameter that characterizes the shape of the rising edge, $\tau_{\text {fall }}$ is the falling time, defined as the time interval between the maximum of the echo and its half-intensity point, i.e., $\sigma^{0}\left(t_{\max }+\tau_{\text {fall }}\right)=\sigma_{\max }^{0} / 2$, and $\beta, 0<\beta<1$, is a further parameter that characterizes the shape of the falling edge.

Fig. 4 shows a sketch of the piecewise function approximating the backscatter profile of the nadir echo, whereas Fig. 5 shows the normalized piecewise function $\left(t_{\max }=0\right.$, $\sigma_{\max }^{0}=1, \tau_{\text {rise }}=1, \tau_{\text {fall }}=1$ ) for different values of $\alpha$ and $\beta$. The values fitting the data acquired over the Indian Ocean and over Australia are: $\tau_{\text {rise }}=28.03$ and $19.48 \mathrm{~ns}, \tau_{\text {fall }}=60.97$ and $6.04 \mathrm{~ns}, \alpha=2.4$ and 1.3 , and $\beta=0.38$ and 0.4 , whereas the ratio of the nadir echo's maximum backscatter to the average backscatter of the background (clutter plus noise) in the range-compressed data is equal to 6.8 and $9.8 \mathrm{~dB}$, respectively. For the dedicated acquisitions the two-way antenna 


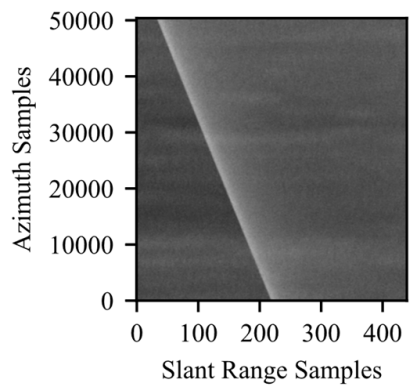

(a)

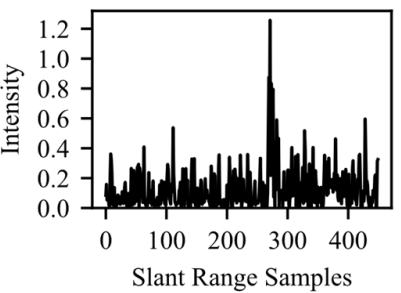

(c)

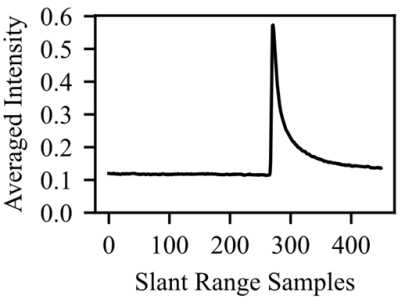

(e)

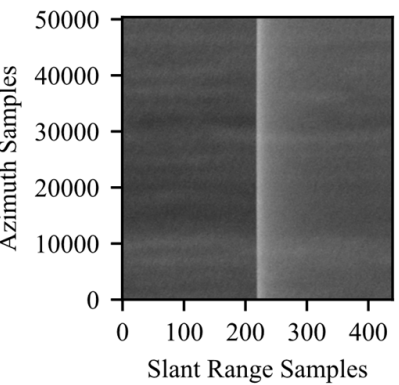

(b)

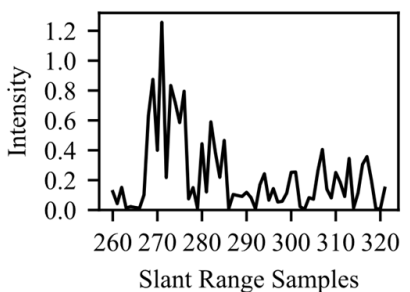

(d)

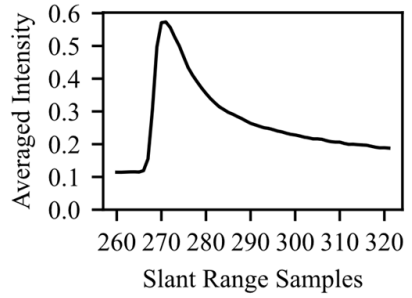

(f)
Fig. 3. Derivation of the nadir backscatter profile from range-compressed data. (a) Portion of range-compressed data affected by nadir echo. (b) Portion of range-compressed data with nadir echoes aligned to the same range. (c) Sample range line of range-compressed data with strong intensity variation due to speckle. (d) Zoom of (b) around the echo peak. (e) Averaged intensity across azimuth. (f) Zoom of (e) around the peak.

pattern attenuation in the nadir direction with respect to the illuminated swath was $50 \mathrm{~dB}$ and the look angle was $26.5^{\circ}$. The value of the nadir echo's maximum backscatter $\sigma_{\max }^{0}$ can be unequivocally defined only after absolute calibration of the data and might better refer to the calibrated focused data to compare it to the backscatter of the rest of the scene. For the dedicated acquisitions, a chirp bandwidth of $150 \mathrm{MHz}$ has been used and a generalized Hamming window with $\alpha=0.6$ has been applied in the processing that leads to a temporal resolution of $8 \mathrm{~ns}$ and a slant range resolution of $1.2 \mathrm{~m}$. A worse range resolution would result in a smoother nadir echo profile [19].

A peculiar characteristic of the nadir echo signal is that its azimuth autocorrelation function is wider than that of the underlying SAR signal for a distributed scatterer. Pulseto-pulse correlation effects in delay/Doppler radar altimetry have been reported in [21] and then exploited for increasing the posting rate in [22]. The azimuth autocorrelation of the underlying SAR signal for a distributed scatterer is given by [9]

$$
\begin{aligned}
R_{\text {signal }}(\xi)= & \left(\frac{v_{s}}{L}\right)^{3}\left[6 \xi^{3} \operatorname{sign}(\xi)+\left(\xi-\frac{L}{v_{s}}\right)^{3}\right. \\
& \times \operatorname{sign}\left(\xi-\frac{L}{v_{s}}\right)+4\left(-\xi+\frac{L}{2 v_{s}}\right)^{3}
\end{aligned}
$$

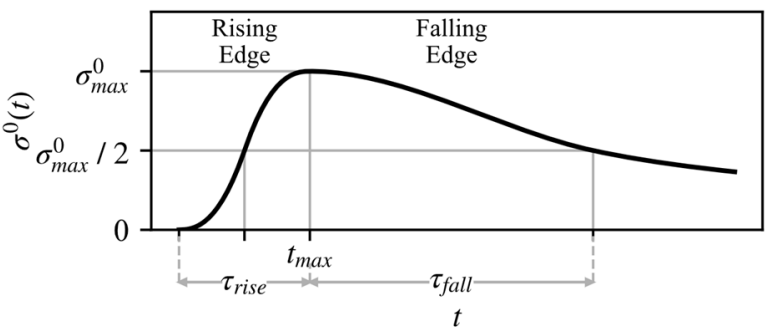

Fig. 4. Piecewise function approximating the backscatter profile of the nadir echo.

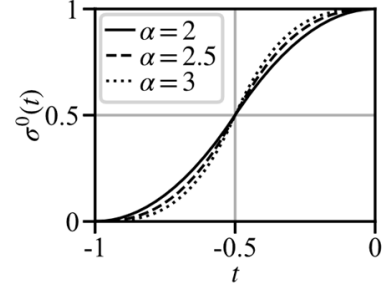

(a)

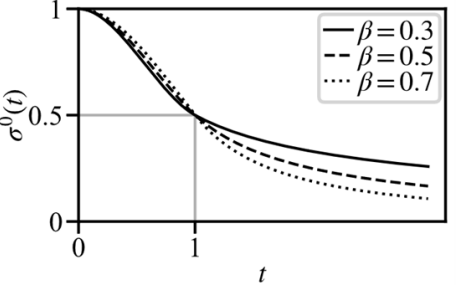

(b)
Fig. 5. (a) Shape of the rising edge for the normalized piecewise function and different values of $\alpha$. (b) Shape of the falling edge for the normalized piecewise function and different values of $\beta$.

$$
\begin{aligned}
& \times \operatorname{sign}\left(\xi-\frac{L}{2 v_{s}}\right)-4\left(\xi+\frac{L}{2 v_{s}}\right)^{3} \\
& \left.\times \operatorname{sign}\left(\xi+\frac{L}{2 v_{s}}\right)+\left(\xi+\frac{L}{v_{s}}\right)^{3} \operatorname{sign}\left(\xi+\frac{L}{v_{s}}\right)\right]
\end{aligned}
$$

where $\xi$ is the azimuth (or slow) time, $v_{s}$ is the satellite velocity and $L$ is the antenna length. It holds $R_{\text {signal }}\left(\xi=L / 2 v_{s}\right)=$ 0.25 , i.e., the autocorrelation of the signal falls to 0.25 for $\xi=$ $0.31 \mathrm{~ms}$ in the TerraSAR-X case $\left(v_{s}=7675 \mathrm{~m} / \mathrm{s}, L=4.8 \mathrm{~m}\right)$.

The azimuth autocorrelation of the nadir echo is instead modeled as a Gaussian autocorrelation function

$$
R_{\text {nadir }}(\xi)=e^{-\left(\frac{\xi}{1}\right)^{2}}
$$

where $l$ is the correlation length, defined here as value of $\xi$ for which the autocorrelation falls to $1 / e \cong 0.37$. The correlation length $l$ can be estimated from the aligned range compressed data [see Fig. 3(b)] considering the azimuth line where the nadir echo peak occurs, subtracting the autocorrelation component due to the clutter and the noise that can be estimated from the azimuth lines preceding the start of the nadir echo, and fitting the resulting curve to the expression in (3). For the data acquired over the Indian Ocean and over Australia the estimated correlation length is equal to $l=0.46$ and $0.29 \mathrm{~ms}$, respectively. Fig. 6 shows the models of autocorrelation functions for the nadir echo and the underlying SAR signal fit to the data acquired over the Indian Ocean. The azimuth autocorrelation function for the nadir echo will be needed in the following for an accurate determination of the ratio of the nadir echo levels between the constant and the variable PRI cases.

\section{Mapping Limitations Due to Nadir Echoes FOR CONSTANT PRI OPERATION}

Denoting as $h$ the height of the platform, which carries the radar antenna, and assuming a constant PRI, the echo from a 


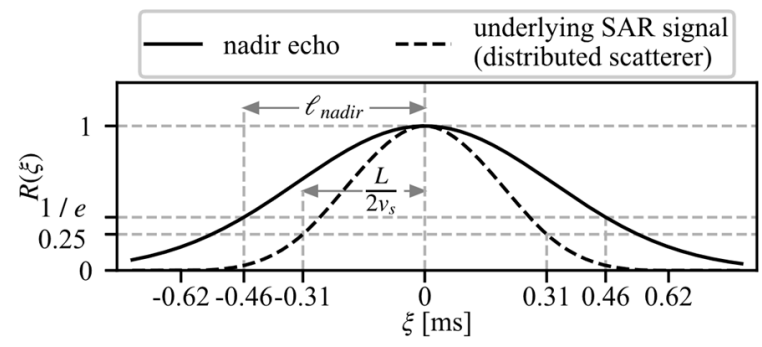

Fig. 6. Models of azimuth autocorrelation functions for the nadir echo and the underlying SAR signal (distributed scatterer) fit to the data acquired over the Indian Ocean.

scatterer at nadir is superimposed on the echo from a scatterer in the scene at slant range $R_{0}$, if the following condition holds:

$$
R_{0}=h+k \frac{c_{0}}{2} \mathrm{PRI}=h+k \frac{c_{0}}{2 \mathrm{PRF}}
$$

where $k$ is an integer number [ $k=2$ in the sketch of Fig. 1(b)], $c_{0}$ is the speed of light in free space, and the pulse repetition frequency (PRF) PRF is the reciprocal of the PRI [23].

In conventional SAR, the nadir interference is usually avoided by choosing a PRF for which the swath echo is received between two consecutive transmitted pulses and the nadir echo comes back at the radar while the radar is transmitting, i.e., neither transmit nor nadir interferences occur [23]. Fig. 7(a) shows the timing (or "diamond") diagram of an SAR, in which for a given PRF (displayed on the $x$-axis) the green zones represent slant ranges (displayed on the $y$-axis) that cannot be imaged due to the transmit interference, i.e., because the radar cannot receive while it is transmitting, whereas the blue zones represent slant ranges that cannot be imaged due to the nadir interference, i.e., the presence of the nadir echo in the imaged scene. PRFs can be therefore chosen for which neither transmit nor nadir interferences occur within the desired swath to be imaged [see red marks in Fig. 7(a)].

In multiple-elevation-beam SAR with constant PRI, a nadir echo will be present for each imaged subswath; therefore, it is not possible to keep the nadir echo out of the imaged area. However, analogously as in conventional SAR, the PRF can be selected in order to have (all) nadir echoes coming back at the radar while the radar is transmitting. In this way, nadir echoes will be superimposed on "blind" areas [Fig. 7(b)].

In particular, the superposition of nadir echoes and blind ranges occurs, if the following condition holds:

$$
\mathrm{PRF} \cong k \frac{c_{0}}{2 h}
$$

where the approximately equal sign is used to account for the temporal (or spatial) extension of nadir echoes and blind ranges. For typical SAR mission orbits, comprised between 500 and $750 \mathrm{~km}$, the PRFs that satisfy the condition in (5) are about $200-300 \mathrm{~Hz}$ apart.

Considering the ambiguity requirements and the data volume constraints, only few distinct PRFs can be selected for which nadir echoes and blind ranges superimpose, e.g., PRFs around 1400, 1600, and $1800 \mathrm{~Hz}$ for the Tandem-L system operating in constant PRI mode, which means that two complete coverages can be achieved every three passes of the satellite. Therefore, while selecting the PRFs according

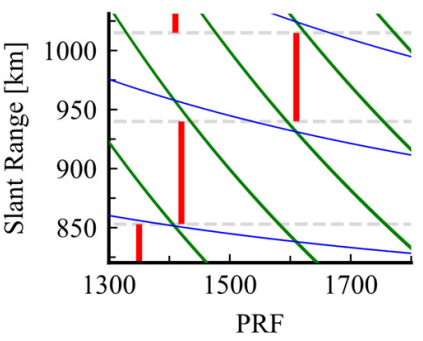

(a)

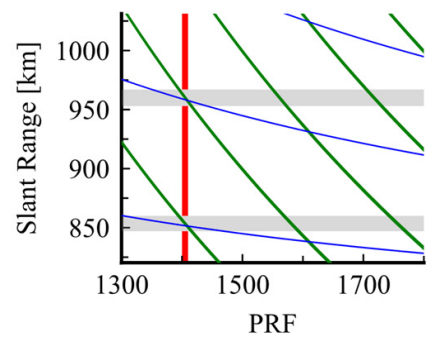

(b)

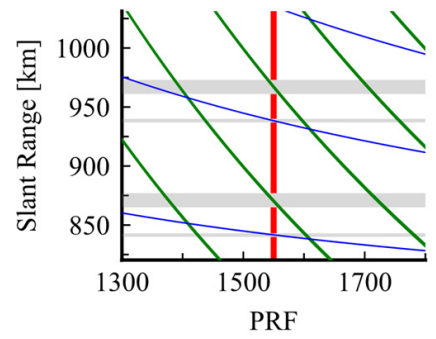

(c)

Fig. 7. Timing (or "diamond") diagrams of (a) a conventional SAR, (b) a multiple-elevation-beam SAR with constant PRI where nadir echoes superimpose on transmit events, and (c) a multiple-elevation-beam SAR, where nadir echoes do not superimpose on transmit events. The green zones represent slant ranges that cannot be imaged due to the transmit interference, whereas the blue zones represent slant ranges that cannot be imaged due to the nadir interference.

to (5) seems to reduce the total useless areas across the swath and minimize the number of subswaths, it might not be the optimal option to uniformly distribute the useless areas across the swath and maximize the number of complete coverages achieved within a given time interval.

If the PRFs are instead selected ignoring the condition in (5), additional useless nadir echo areas are present across the swath in addition to blind ranges [Fig. 7(c)]. In this case, it can be shown that the number of complete coverages achieved within a given time interval depends on the pulse width. Fig. 8(a) and (b) show for the Tandem-L system operating in constant PRI mode the location of nadir echoes (in blue) and blind ranges (in green) across the swath for different passes (and corresponding distinct PRFs within the interval $1350-1840 \mathrm{~Hz}$ ) for pulse width equal to 15 and $45 \mu \mathrm{s}$, respectively. Seven complete coverages can be achieved every eight passes in the first case, whereas only three complete coverages every four passes in the second case.

A pulse width of $15 \mu \mathrm{s}$ corresponds to a duty cycle in the order of $2 \%$, which is not ideal to guarantee the required noise-equivalent sigma zero under typical peak power constraints. Therefore, even in case nadir echoes and blind ranges do not superimpose, the ratio between the number of complete coverages to the number of passes is not likely to be higher than $75 \%-80 \%$.

\section{Formulation OF NADIR ECHO LOCATION IN STAGGERED SAR DATA}

A multiple-elevation-beam SAR is operated in staggered SAR mode if the PRI is varied from pulse to pulse. As a consequence of the continuous PRI variation, the blind ranges will be different for each transmitted pulse and, as long as the 


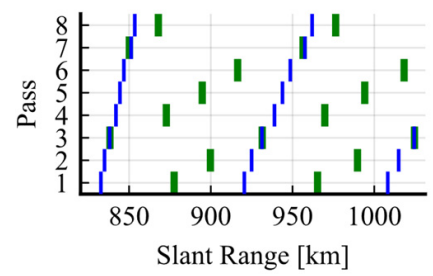

(a)

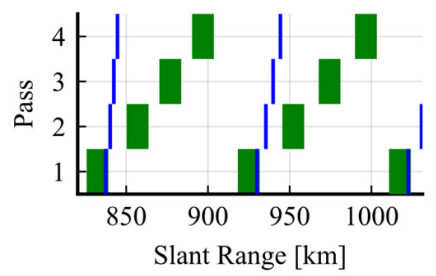

(b)
Fig. 8. Location of nadir echoes (in blue) and blind ranges (in green) across the swath for different passes (and corresponding distinct PRFs) for the Tandem-L system operating in constant PRI mode. (a) Pulse width equal to $15 \mu$ s. Seven complete coverages can be achieved every eight passes. (b) Pulse width equal to $45 \mu \mathrm{s}$. Three complete coverages can be achieved every four passes.

sequence of PRIs is properly selected and data are moderately oversampled in azimuth, the non-uniformly sampled, gapped raw data can be interpolated onto a uniform grid and focused with a conventional SAR processor to obtain a high-resolution SAR image over a wide continuous swath [9]-[12].

Under the assumption that a sequence of $M$ distinct PRIs, $\mathrm{PRI}_{k}, k=0, \ldots, M-1$, is employed and repeats periodically, the location of the blind ranges in the raw data can be described through the blockage diagram. Fig. 9 illustrates the construction of the blockage diagram for the simplified case of a sequence of $M=6$ PRIs: On the top part of the figure the transmitted pulses separated by varying PRIs are displayed on a time axis, where the circled number indicates the pulse index; immediately below, the received echoes corresponding to the transmitted pulses are shown assuming that echo recording starts as the pulse begins to be transmitted (the circled number on the left of the echoes always indicates the pulse index); in the bottom part of the figure, the blockage diagram, obtained by aligning all received echoes, is shown for a single cycle of PRI variation, as it repeats then periodically, and as a function of slant range $R$, where $R$ is related to fast time through a scaling factor $c_{0} / 2$.

For nonnegative pulse indices $k, k \geq 0$, the blind ranges start at $R_{\text {blind } k, \rho}$ given by

$$
R_{\text {blind }, \rho}=\frac{c_{0}}{2} \sum_{n=k}^{k+\rho-1} \operatorname{PRI}_{n \bmod M}, \rho \geq 0
$$

where mod is the modulo operator and $\rho$ is here defined as the "ambiguity order," i.e., echoes of transmitted pulse $k$ cannot be received from range $R_{\text {blind } k, \rho}$, because pulse $k+\rho$ is being transmitted. $R_{\text {blind } k, \rho}$ is therefore given by a moving sum of $\rho$ consecutive PRIs, starting with $\mathrm{PRI}_{k \bmod M}$, times $c_{0} / 2$. As an example, $R_{\text {blind } 2,3}$ is marked in Fig. 9, where it is also apparent that the start of the blind range is given by the sum of three consecutive PRIs (as $\rho=3$ in this example) starting with $\mathrm{PRI}_{2}$ (as $k \bmod M=2 \bmod 6=2$ in this example) times $c_{0} / 2$, and where blind range intervals with the same ambiguity order $\rho$ are shown using the same color. Furthermore, blind ranges extend over a slant range interval given by the uncompressed pulse width $\tau$ times $c_{0} / 2$.

While in Fig. 9 the blockage diagram is shown for the sake of explanation for slant ranges starting at $R=0$, only slant ranges within the imaged swath, i.e., $R_{\min } \leq R \leq R_{\max }$, are of practical interest, and the sequence of PRIs of a staggered
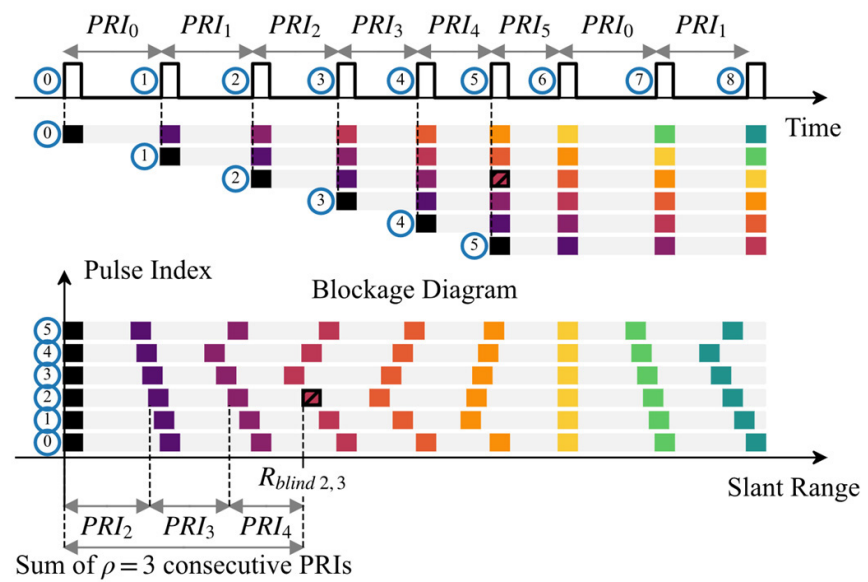

Fig. 9. Construction of the blockage diagram. Transmitted pulses separated by varying PRIs, received echoes corresponding to the transmitted pulses, and resulting blockage diagram as a function of slant range.

SAR is indeed designed so that two consecutive azimuth samples are never missed in the raw data within the imaged swath [11]. Fig. 10 shows an exemplary blockage diagram for the staggered SAR system of the Tandem-L mission proposal, where 233 distinct PRIs are used and a 211-km slant range swath (corresponding to a $350-\mathrm{km}$ ground swath) is imaged.

Analogously to the blockage diagram, a nadir echo location diagram can be constructed, where, for each pulse index, the slant ranges where the nadir echo is expected to appear are identified [27]. While the blockage diagram is utilized to verify that for a given PRI sequence two consecutive azimuth samples are never missed in the raw data within the swath of interest, the nadir echo location diagram helps characterizing the expected nadir echo signature. For this reason, while the blockage diagram refers to the raw data, as it is better to resample data before range-compression [10], it is more convenient to construct a nadir echo location diagram that refers to range-compressed data.

Fig. 11 illustrates the construction of the nadir echo location diagram for the previously shown, simplified case of a sequence of $M=6$ PRIs: On the top part of the figure the transmitted pulses separated by varying PRIs are displayed on a time axis; immediately below, the nadir echoes corresponding to each of the transmitted pulses and the full received echoes including nadir echoes are shown; in the bottom part of the figure, the nadir echo location diagram, obtained by aligning all received echoes, is finally shown. The slant ranges affected by nadir echoes start at $R_{\text {nadirk, } \rho}$ given by

$$
\begin{aligned}
R_{\text {nadirk }, \rho} & =h+\frac{c_{0}}{2} \sum_{n=k}^{k+\rho-1} \operatorname{PRI}_{n \bmod M} \\
& =h+R_{\text {blind } k, \rho}, \quad \rho \geq 0 .
\end{aligned}
$$

$R_{\text {nadirk, } \rho}$ is therefore the sum of $h$ and a moving sum of $\rho$ consecutive PRIs, starting with $\mathrm{PRI}_{k \bmod M}$, times $c_{0} / 2$ and, if the pulse width and the extension of the nadir echo are ignored, the nadir echo location diagram corresponds to the blockage diagram horizontally translated by a quantity given by the orbit height $h$. 


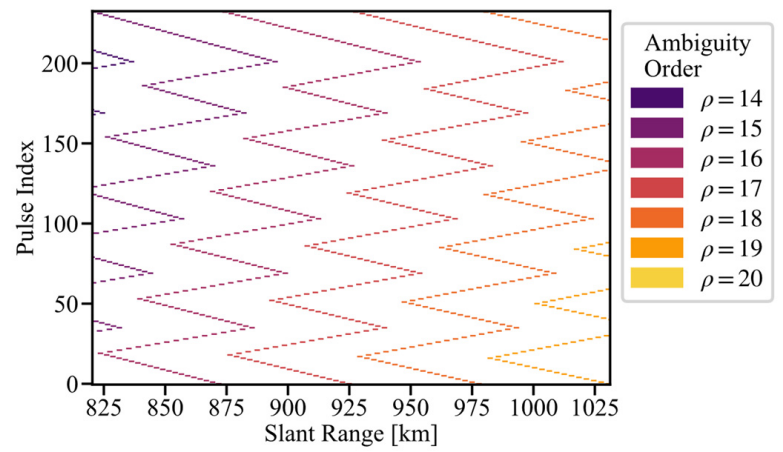

Fig. 10. Exemplary blockage diagram for the staggered SAR system of the Tandem-L mission proposal. Blind range intervals with the same ambiguity order $\rho$ are shown using the same color.

In practice, if the nadir echo location diagram is restricted to the swath of interest, it appears quite different from the blockage diagram, as it can be noticed by comparing Figs. 10 and 12, where the nadir echo location diagram for the staggered SAR system of the Tandem-L mission proposal is shown. The reason is that the visible ambiguity orders in the nadir echo location diagram are much smaller than those visible in the blockage diagram, and the slant range intervals where nadir echoes with a given ambiguity order extend over are usually separated from each other rather than overlapping (and highlighted as semi-transparent regions in Fig. 12). The center slant range $R_{\text {nadirmean } \rho}$ of the latter intervals is given by

$$
\begin{aligned}
R_{\text {nadirmean } \rho} & =h+\rho \frac{c_{0}}{2} \sum_{n=0}^{M-1} \mathrm{PRI}_{n} \\
& =h+\rho \frac{c_{0}}{2} \mathrm{PRI}_{\text {mean }}, \rho \geq 0
\end{aligned}
$$

where PRI mean denotes the average PRI of the sequence, whereas the upper bound of the extension of the intervals $\Delta R_{\text {nadir } \rho}$ (always neglecting the nadir echo extension) is given by

$$
\Delta R_{\text {nadir } \rho}=\rho \frac{c_{0}}{2}\left(\mathrm{PRI}_{\max }-\mathrm{PRI}_{\min }\right), \quad \rho \geq 0
$$

where $\mathrm{PRI}_{\max }$ and $\mathrm{PRI}_{\min }$ denote the maximum and minimum PRI of the sequence.

The extension of the latter intervals is very important to assess the range interval over which the nadir echo energy spreads with respect to the constant PRI case. The worst case (smaller interval) occurs, as is also apparent from the example of Fig. 12, at near range and for the smallest ambiguity order within the swath, denoted as $\rho_{\min }$. Under the assumption that the $\rho_{\min }$ maximum PRIs of the sequence are consecutive and almost equal to $\mathrm{PRI}_{\max }, \rho_{\min }$ is given by

$$
\rho_{\min }=\left\lceil\frac{\left(R_{\min }-h\right)}{\mathrm{PRI}_{\max }} \frac{2}{c_{0}}\right\rceil
$$

where $\lceil\cdot\rceil$ is the ceil operator, and the worst case for the slant range interval over which the nadir echo $\Delta R_{\text {nadir } \rho_{\min }}$ is smeared is approximately given by

$$
\begin{aligned}
\Delta R_{\text {nadir } \rho_{\min }} & \cong \rho_{\min } \frac{c_{0}}{2}\left(\mathrm{PRI}_{\max }-\mathrm{PRI}_{\min }\right) \\
& \cong\left\lceil\frac{\left(R_{\min }-h\right)}{\mathrm{PRI}_{\max }} \frac{2}{c_{0}}\right\rceil \frac{c_{0}}{2}\left(\mathrm{PRI}_{\max }-\mathrm{PRI}_{\min }\right)
\end{aligned}
$$
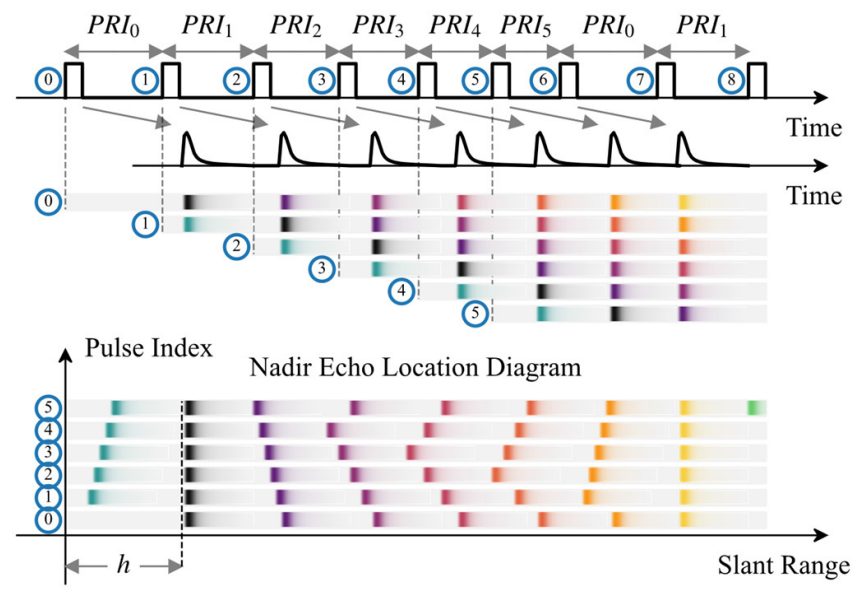

Fig. 11. Construction of the nadir echo location diagram. Transmitted pulses separated by varying PRIs, nadir echoes for the transmitted pulses, received echoes corresponding to the transmitted pulses including all nadir echoes, and resulting nadir echo location diagram as a function of slant range.

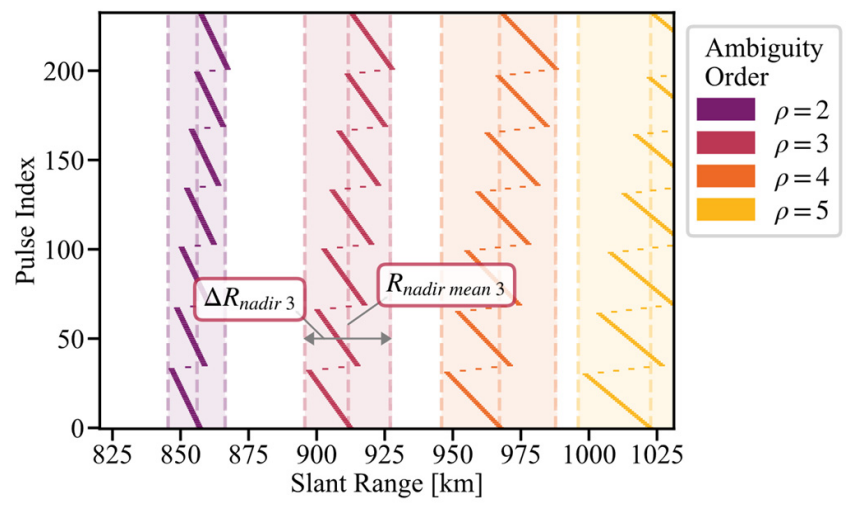

Fig. 12. Exemplary nadir echo location diagram for the staggered SAR system of the Tandem-L mission proposal. Slant range affected by nadir echoes with the same ambiguity order $\rho$ are shown using the same color.

In the Tandem-L example presented in this article, in the worst case (near range) the nadir echo energy spreads over a slant range interval $\Delta R_{\text {nadir }_{\text {min }}} \cong 20.6 \mathrm{~km}$, which corresponds to almost two thousand times the slant range extension in the case of constant PRI (about $11 \mathrm{~m}$, assuming it equal to $c_{0}\left(\tau_{\text {rise }} / 2+\tau_{\text {fall }}\right) / 2$ and substituting the values provided in Section II). For the nadir echo energy to spread in an as uniform as possible way over the $\Delta R_{\text {nadir } \rho_{\text {min }}}$ interval, the number of PRIs of the sequence $M$ should be at least in the order of the ratio of the slant range interval $\Delta R_{\text {nadir } \rho_{\min }}$ to the slant range extension in the case of constant PRI (note that in the Tandem-L example $M=233$ is lower than the aforementioned ratio, as the developments of this work were not taken into account within the design). A very long sequence of PRIs, where two consecutive samples are never missed in the raw data along azimuth, can be obtained following the design principles for more elaborated sequences described in [11], [12].

In principle, the PRI sequence can be selected to spread the nadir echo energy over a larger interval by extending the PRI span. However, this has a negative impact on the level of range and/or azimuth ambiguities and therefore on the image quality. 


\section{Simulation ExAmples BASEd ON TERRASAR-X DATA}

The nadir echo location diagram refers to the rangecompressed data and one cycle of PRI variation. As azimuth compression is performed to obtain a focused SAR image, smearing of the nadir echoes in the azimuth direction occurs. In order to get some examples of nadir echo responses in the focused SAR image, some realistic simulations based on TerraSAR-X data are carried out.

Starting from a portion of a complex focused SAR image acquired by the TerraSAR-X satellite in stripmap mode and assuming the complex backscatter of the imaged region is given by the focused SAR image itself, nadir-echo-free raw staggered SAR data are simulated over a part of the imaged swath by introducing a range and azimuth modulation according to the system parameters of the Tandem-L example [14]. As the pixel spacings of the simulated system and of the original image are different, the distances in the simulated data are not exactly the same as in the reality. Furthermore, nadir echoes are simulated according to the backscatter profile model provided in Section II, and, after having introduced a range modulation, located in the raw data matrix according to the formulation of Section IV. Fig. 13 shows some portions of the raw staggered SAR data and of the focused data for the underlying nadir-echo-free scene and for the nadir echo.

The same simulation has also been carried for the constant PRI case and, following the considerations on the number of PRIs of the sequence at the end of Section IV, for a staggered SAR case with the same parameters of the Tandem-L example, but with a longer sequence of PRIs, i.e., $M=1000$.

A comparison of how the nadir echoes corrupt the underlying scene in multiple-elevation-beam SAR with constant PRI and staggered SAR is shown in Fig. 14, having assumed the same nadir echo backscatter profile. To observe and compare the nadir echo signatures a very strong, unrealistic, nadir echo is considered, characterized by the largest observed width and a nadir-to-clutter-plus-noise ratio in the range-compressed data equal to $30 \mathrm{~dB}$, i.e., more than 23 and $20 \mathrm{~dB}$ higher than that of the images in Fig. 2 acquired over Australia and the Indian Ocean, respectively, and $7 \mathrm{~dB}$ higher than the worst possible case obtained if the land nadir echo of the image acquired over Australia had superimposed on the background of the image acquired over the Indian Ocean. The resulting focused images are shown using a 36-dB logarithmic scale. Even considering this huge margin, which allow accounting for the variability of the responses from different surfaces and at different wavelengths, in the staggered SAR case, the smeared nadir echo shows a striped pattern for $M=233$ and a noise-such as pattern for $M=1000$, and is barely visible even over the calm water surface characterized by very low backscatter such that it is likely to be well below the thermal noise level in typical cases.

Fig. 15 displays the nadir echo energy integrated over azimuth as a function of slant range for the three scenarios of Fig. 14, from which it is possible to observe that the nadir echo level for the staggered SAR case with $M=$ 1000 is about $26 \mathrm{~dB}$ lower than for the constant PRI case. For sufficiently large $M$, a rough estimate of the nadir echo level
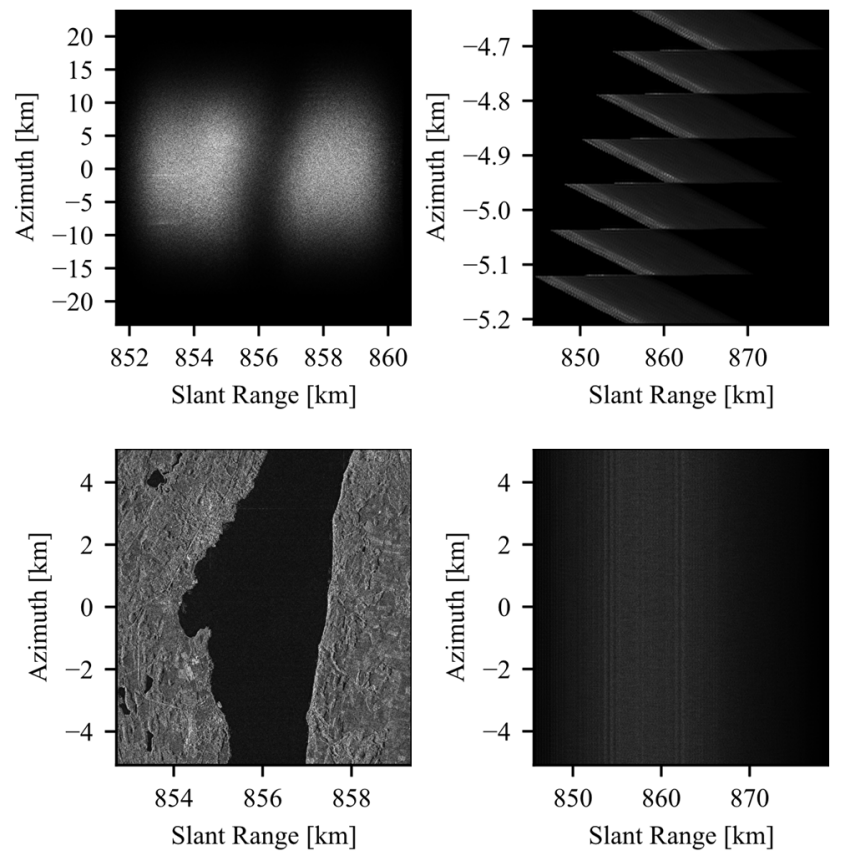

Fig. 13. Portions of simulated staggered SAR raw data (top) and focused data (bottom) for the nadir-echo-free underlying scene (left) and the nadir echoes (right). Different range and azimuth scales are used for the different quadrants.

in staggered SAR relative to the constant PRI case is given by the smearing factor computed at the end of Section IV. While this estimate does not account for the nonuniform resulting smearing for typical more elaborated sequences of PRIs (see green curve in Fig. 15) and the incoherent integration in azimuth of the nadir echo energy, which spreads almost uniformly across the Doppler spectrum in the staggered SAR case and, as the mean PRF of the system is significantly larger than the processed Doppler bandwidth, is partly filtered out during the SAR processing (especially if a Hamming window is used), these factors compensate each other and could be neglected.

Despite the intrinsic nadir echo smearing provided by the staggered SAR mode, the radar antenna has to guarantee a significant suppression in the nadir direction in order to avoid saturation of the receiver. For the Tandem-L design the two-way antenna pattern attenuation at nadir ranges between 75 and $90 \mathrm{~dB}$ with an attenuation on transmit of around $40 \mathrm{~dB}$, although the real values could be worse, due to e.g., the sidelobes resulting from the quasi-flat facets of the mesh reflector [25].

One option to control the gain at nadir is to place a notch in the antenna pattern. As staggered SAR systems are likely to be implemented using large deployable parabolic reflectors, illuminated by digital feed arrays with multiple receive channels [14], [15], a precise knowledge of the complex secondary far-field antenna patterns associated with the excitation of each single-feed element is required for that. As it is impossible to measure these antenna patterns prior to the launch with sufficient accuracy, the novel technique for highly-accurate in-orbit measurement of the relative phase and amplitude of 


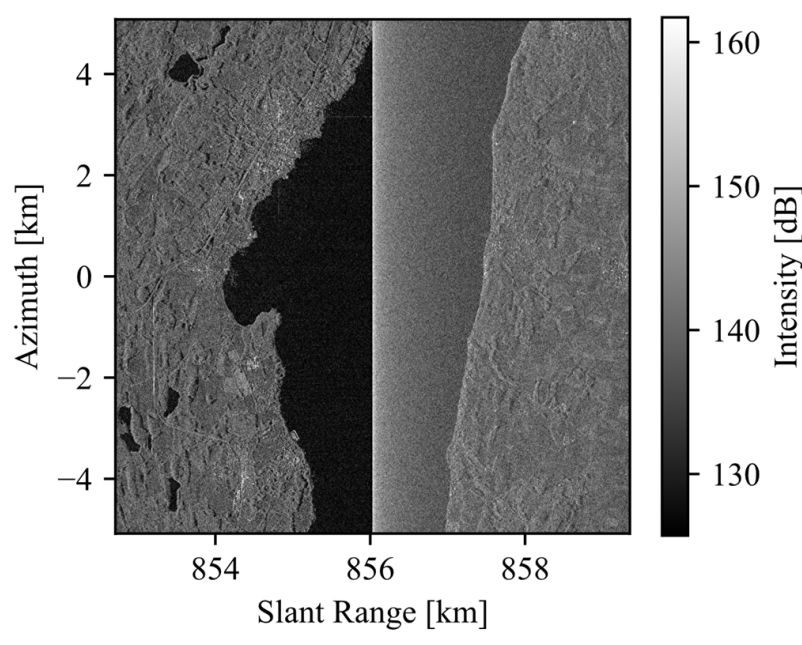

(a)

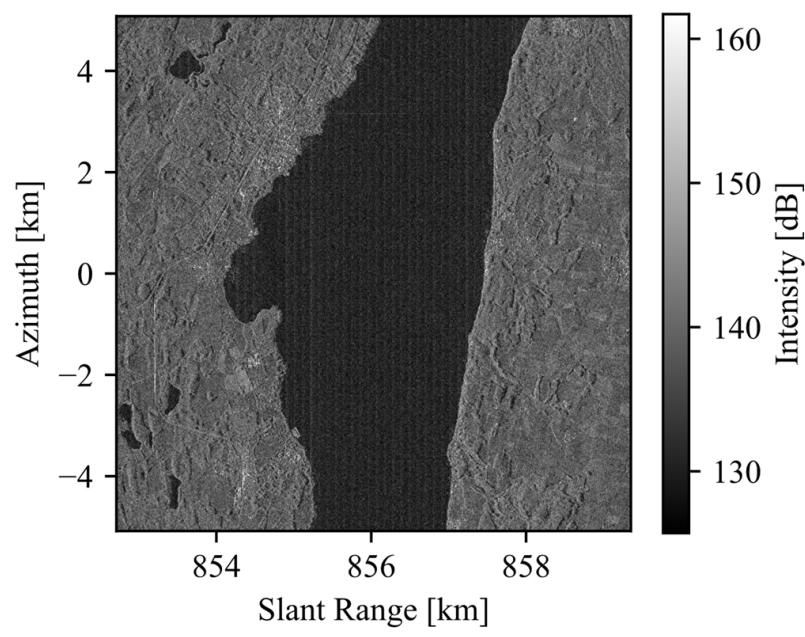

(b)

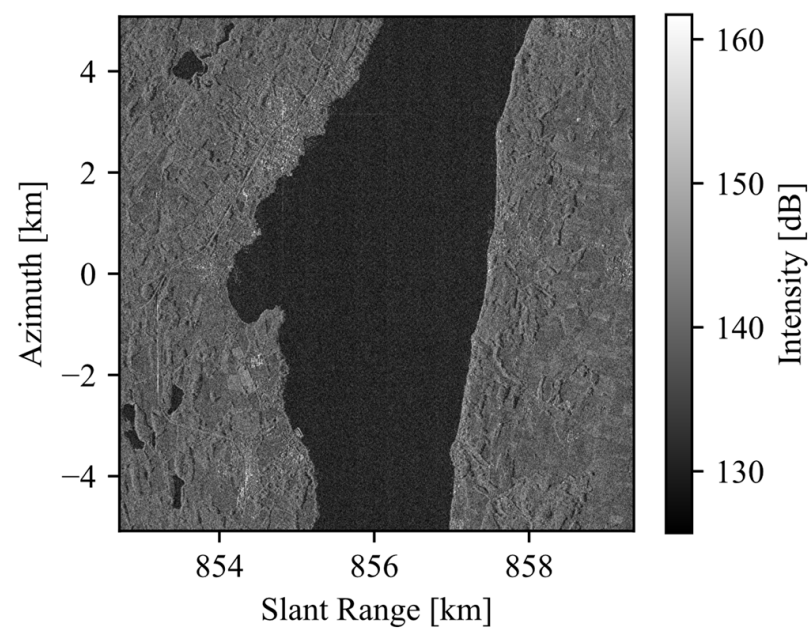

(c)

Fig. 14. Effect of a very strong nadir echo on simulated multipleelevation-beam SAR images. (a) Constant PRI. (b) Staggered SAR with $M=233$ PRIs. (c) Staggered SAR with $M=1000$ PRIs.

the far-field secondary antenna patterns from individual feed elements in both elevation and azimuth presented in [26] can be exploited.

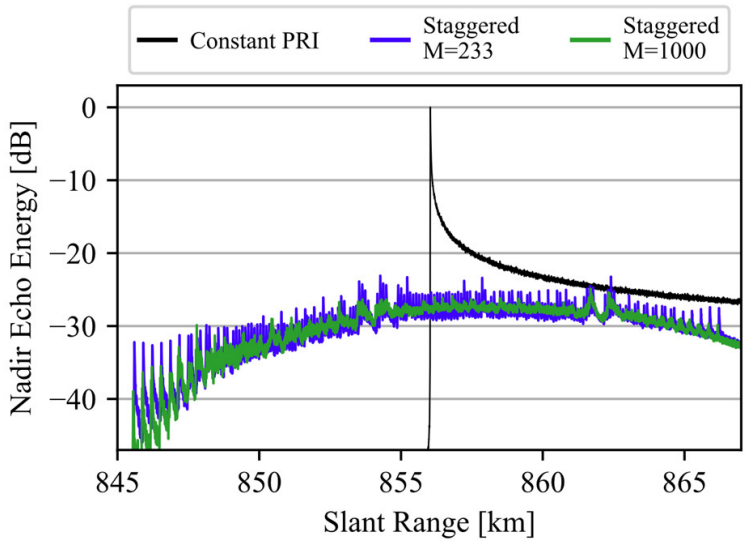

Fig. 15. Nadir echo energy integrated over azimuth as a function of slant range for the scenarios of Fig. 14 (a) (black), (b) (Blue), and (c) (green).

\section{CONCLUSION AND OUTLOOK}

This article extends the theory of multiple-elevation-beam SAR with constant and variable PRI by describing how nadir echoes affect the mapping capability for constant PRI operation and the image quality for staggered SAR.

In particular, it is shown that for constant PRI operation the ratio between the number of complete coverages to the number of passes is not likely to be higher than $75 \%-80 \%$ for typical scenarios, whereas a detailed theoretical formulation of nadir echo location in staggered SAR data is presented that allows assessing over which slant range intervals nadir echoes are located and how long the sequence of PRI should be in order for the nadir echo energy to spread in an as uniform as possible way over those intervals. A simulation example based on TerraSAR-X data confirms the theoretical results and shows a scenario where a specific staggered SAR system the nadir echo level is about $26 \mathrm{~dB}$ lower than for the constant PRI case. The nadir echo is barely visible even assuming a huge margin on the nadir-to-clutter-plus-noise ratio in the range-compressed data that allow accounting for the variability of the responses from different surfaces and at different wavelengths.

The results of this work are relevant to the design of future SAR systems and the interpretation of the acquired data. This work also represents a starting point to devise and elaborate processing methods for nadir echo suppression in staggered SAR. The knowledge of the nadir echo location in range-compressed staggered SAR data, in fact, allows identifying for each range line of range-compressed data which samples are affected by nadir echoes. As nadir echoes are located at different ranges for different range lines and staggered SAR data are characterized by moderate azimuth oversampling, samples affected by nadir echoes could be replaced by values obtained from interpolation of neighboring azimuth samples exactly as done for missing data due to the transmit interference. This could be implemented as an optional additional stage in the SAR processing chain and used to reprocess raw data in case some smeared nadir echo is still visible and critical for some specific applications [27].

\section{ACKNOWLEDGMENT}

The authors would like to thank their colleagues Dr. Muriel Pinheiro, Prof. Gerhard Krieger, and 
Prof. Alberto Moreira for many constructive discussions and the anonymous reviewers for their helpful comments.

\section{REFERENCES}

[1] A. Moreira, P. Prats-Iraola, M. Younis, G. Krieger, I. Hajnsek, and K. P. Papathanassiou, "A tutorial on synthetic aperture radar," IEEE Geosci. Remote Sens. Mag., vol. 1, no. 1, pp. 6-43, Mar. 2013.

[2] A. Currie and M. A. Brown, "Wide-swath SAR," IEE Proc. F Radar Signal Process., vol. 139, no. 2, pp. 122-135, Apr. 1992.

[3] G. D. Callaghan and I. D. Longstaff, "Wide-swath space-borne SAR using a quad-element array," IEE Proc.-Radar, Sonar Navigat., vol. 146, no. 3, pp. 159-165, 1999 .

[4] M. Suess, B. Grafmueller, and R. Zahn, "A novel high resolution, wide swath SAR system," in Proc. Scanning Present Resolving Future IEEE Int. Geosci. Remote Sens. Symp. (IGARSS), Jul. 2001, pp. 1013-1015.

[5] G. Krieger, N. Gebert, and A. Moreira, "Unambiguous SAR signal reconstruction from nonuniform displaced phase center sampling," IEEE Geosci. Remote Sens. Lett., vol. 1, no. 4, pp. 260-264, Oct. 2004.

[6] N. Gebert, G. Krieger, and A. Moreira, "Digital beamforming on receive: Techniques and optimization strategies for high-resolution wide-swath SAR imaging," IEEE Trans. Aerosp. Electron. Syst., vol. 45, no. 2, pp. 564-592, Apr. 2009.

[7] G. Krieger, N. Gebert, M. Younis, F. Bordoni, A. Patyuchenko, and A. Moreira, "Advanced concepts for ultra-wide-swath SAR imaging," in Proc. EUSAR, Friedrichshafen, Germany, Jun. 2008, pp. 1-4.

[8] A. Freeman et al., "SweepSAR: Beam-forming on receive using a reflector-phased array feed combination for spaceborne SAR," in Proc. IEEE Radar Conf., Pasadena, CA, USA, May 2009, pp. 1-9.

[9] M. Villano, G. Krieger, and A. Moreira, "Staggered SAR: Highresolution wide-swath imaging by continuous PRI variation," IEEE Trans. Geosci. Remote Sens., vol. 52, no. 7, pp. 4462-4479, Jul. 2014.

[10] M. Villano, G. Krieger, and A. Moreira, "A novel processing strategy for staggered SAR," IEEE Geosci. Remote Sens. Lett., vol. 11, no. 11, pp. 1891-1895, Nov. 2014.

[11] M. Villano, "Staggered synthetic aperture radar," Ph.D. dissertation, Karlsruhe Inst. Technol., DLR-Forschungsbericht Wessling, Karlsruhe, Germany, 2016.

[12] M. Villano, G. Krieger, M. Jäger, and A. Moreira, "Staggered SAR Performance analysis and experiments with real data," IEEE Trans. Geosci. Remote Sens., vol. 55, no. 11, pp. 6617-6638, Nov. 2017.

[13] A. Moreira et al., "Tandem-L: A highly innovative bistatic SAR mission for global observation of dynamic processes on the earth's surface," IEEE Geosci. Remote Sens. Mag., vol. 3, no. 2, pp. 8-23, Jun. 2015.

[14] S. Huber, F. Q. de Almeida, M. Villano, M. Younis, G. Krieger, and A. Moreira, "Tandem-L: A technical perspective on future spaceborne SAR sensors for earth observation," IEEE Trans. Geosci. Remote Sens., vol. 56, no. 8, pp. 4792-4807, Aug. 2018.

[15] P. A. Rosen et al., "Impact of gaps in the NASA-ISRO SAR mission swath," in Proc. 12th Eur. Conf. Synth. Aperture Radar (EUSAR), Aachen, Germany, Jun. 2018, pp. 1-5.

[16] M. Villano et al., "Gapless imaging with the NASA-ISRO SAR (NISAR) mission: Challenges and opportunities of staggered SAR," in Proc. Eur. Conf. Synth. Aperture Radar (EUSAR), Aachen, Germany, Jun. 2018, pp. 1-6.

[17] T. Motohka, Y. Kankaku, S. Miura, and S. Suzuki, "Alos-4 L-band SAR mission and observation," in Proc. IGARSS - IEEE Int. Geosci. Remote Sens. Symp., Yokohama, Japan, Jul. 2019, pp. 5271-5273.

[18] M. Zonno, J. Matar, F. Queiroz de Almeida, M. Rodriguez Cassola, and G. Krieger, "Sentinel-1 next generation: Trade-offs and assessment of mission performance," in Proc. ESA Living Planet Symp., Milan, Italy, May 2019.

[19] R. K. Raney, "The delay/Doppler radar altimeter," IEEE Trans. Geosci. Remote Sens., vol. 36, no. 5, pp. 1578-1588, Sep. 1998.

[20] C. Ray et al., "SAR altimeter backscattered waveform model," IEEE Trans. Geosci. Remote Sens., vol. 53, no. 2, pp. 911-919, Feb. 2015.

[21] A. Egido and W. H. F. Smith, "Pulse-to-pulse correlation effects in high PRF low-resolution mode altimeters," IEEE Trans. Geosci. Remote Sens., vol. 57, no. 5, pp. 2610-2617, May 2019.

[22] A. Egido, S. Dinardo, and C. Ray, "The case for increasing the posting rate in delay/Doppler altimeters," Adv. Space Res., vol. 68, no. 2, pp. 930-936, Jul. 2021.

[23] J. C. Curlander and R. N. McDonough, Synthetic Aperture Radar: Systems and Signal Processing. New York, NY, USA: Wiley, 1991.
[24] J. Balkowski and F. Bordoni, "Nadir echo properties, a study based on TerraSAR-X data," in Proc. TELFOR, Belgrade, Serbia, Nov. 2012, pp. $420-423$.

[25] J. Angevain, G. Rodrigues, J. Santiago-Prowald, C. Mangenot, and L. Datashvili, "Phyllotactic arrangements of reflector mesh facets to decrease grating lobes," in Proc. 9th Eur. Conf. Antennas Propag. (EuCAP), Apr. 2015, pp. 1-5.

[26] G. Krieger et al., "In-orbit relative amplitude and phase antenna pattern calibration for tandem-L," in Proc. Eur. Conf. Synth. Aperture Radar (EUSAR), Aachen, Germany, Jun. 2018, pp. 1-6.

[27] M. Nogueira Peixoto, M. Villano, and G. Krieger, "Nadir echo suppression in staggered SAR," in Proc. Adv. RF Sensors Remote Sens. Instrum., Noordwijk, The Netherlands, Nov. 2019.

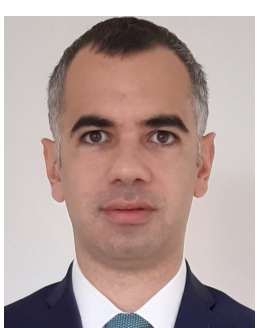

Michelangelo Villano (Senior Member, IEEE) received the B.Sc. and M.Sc. degrees (Hons.) in telecommunication engineering from the Sapienza University of Rome, Rome, Italy, in 2006 and 2008, respectively, and the Ph.D. degree (Hons.) in electrical engineering and information technology from Karlsruhe Institute of Technology, Karlsruhe, Germany, in 2016.

From 2008 to 2009, he was a Young Graduate Trainee with the European Space Research and Technology Center, European Space Agency, Noordwijk, The Netherlands, where he developed processing algorithms for ice sounding radar. In 2017, he was a Visiting Research Scientist with the Communications, Tracking, and Radar Division, NASA Jet Propulsion Laboratory, Pasadena, CA, USA, where he analyzed novel acquisition modes for the NASA Indian Space Research Organization synthetic aperture radar (SAR) instrument. Since 2009, he has been with the German Aerospace Center (DLR), Microwaves and Radar Institute, Wessling, Germany, where he is currently the Head of the NewSpace SAR Research Group. Since 2019, he has also been a Lecturer with Ulm University, Ulm, Germany. He has authored or coauthored over 90 research articles in peer-reviewed journals and international conference proceedings. He holds nine patents in the field of SAR. His research interests include the conception of innovative SAR modes for high-resolution wide-swath imaging and the development of low-cost SAR solutions for frequent and enhanced Earth monitoring.

Dr. Villano has been a member of the Technical Program Committee of the European Conference on SAR (EUSAR) since 2016. He was a recipient of the First Place Student Paper Award at the EUSAR, Berlin, Germany, in 2014, the IEEE Geoscience and Remote Sensing Society Letters Prize Paper Award in 2015 and 2017, the Student Paper Award at the Asia-Pacific Conference on Synthetic Aperture Radar, Marina Bay Sands, Singapore, in 2015, the DLR Science Award in 2016, the Award as Young Scientist of the Foundation Werner von Siemens Ring in 2017, the Informationstechnische Gesellschaft [Information Technology Society (ITG)] Dissertation Award in 2017, and the Best Paper Award at the German Microwave Conference 2019. He is the Co-Chair of the Working Group on Remote Sensing Instrument and Technologies for Small Satellites of the IEEE Geoscience and Remote Sensing Society's Technical Committee on Instrumentation and Future Technologies. He serves as an Associate Editor for the IEEE GEOSCIENCE AND REMOTE SEnSING LetTers. He has served as a Guest Editor for the Special Issues Advances in Antenna Array Processing for Radar 2014 and Advances in Antenna Array Processing for Radar 2016 of the International Journal of Antennas and Propagation.

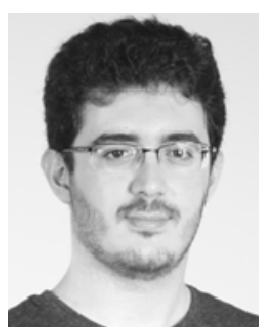

Maxwell Nogueira Peixoto was born in Feira de Santana-BA, Brazil, in 1996. He received the B.S. degree in electronic engineering and the M.S. degree in telecommunications from the Aeronautics Institute of Technology (ITA), São José dos Campos-SP, Brazil, in 2020 and 2021, respectively.

In 2019, he was an Intern at the Microwaves and Radar Institute of the German Aerospace Center (DLR), Wessling, Germany, where he worked in the NewSpace SAR Research Group on the topic of nadir echo characterization and suppression in staggered SAR. His research interests include radar signal processing, staggered SAR, and multibaseline SAR interferometry. 